S yphilis, caused by infection with Treponema pallidum subspecies pallidum, is a mucocutaneous sexually transmitted infection (STI) with high infectivity in the early infectious stages. It may also be passed transplacentally from the ninth week of gestation onwards. The primary stage, with an incubation period of 9-90 days, usually consists of a painless single ulcer at the site of inoculation that may be accompanied by regional lymphadenopathy. The secondary stage, with an incubation period of 6 weeks to 6 months, has many clinical manifestations including rash, mouth ulcers, condylomata lata, patchy alopecia, generalised lymphadenopathy, meningitis, and hepatitis. The later, non-infectious, tertiary stage manifestations of syphilis are now rarely seen in the United Kingdom and include gummatous syphilis, neurosyphilis, and cardiovascular syphilis. Latent syphilis may be divided into early (less than 2 years' duration) and late (more than 2 years' duration).

Screening is recommended for all asymptomatic patients attending a UK genitourinary medicine (GUM) clinic.

There are no controlled studies to support this statement but the recent increase in infectious syphilis in the United Kingdom and other European countries ${ }^{1}$ supports screening as part of good clinical practice. Apart from the public health benefit of detecting infectious syphilis, screening will detect non-infectious stages of syphilis, which will benefit the individual patient.

Patients with syphilitic lesions will require further investigation as outlined below.

\section{RECOMMENDED TESTS}

\section{Serological screening tests}

Treponema pallidum enzyme immunoassay (EIA) (evidence level IIb; recommendation grade B)

There are a number of different EIAs to detect antitreponemal antibodies and very few have been subject to peer review evaluation so it is important to establish satisfactory performance of any EIA used; this applies to all types of serological test.

EIAs that detect both IgG and IgM are recommended as they tend to be more sensitive in primary infection ${ }^{23}$ (evidence level IIb; recommendation grade B).

The Treponema pallidum particle assay (TPPA) is recommended in preference to the Treponema pallidum haemagglutination assay (TPHA) ${ }^{4}$ (evidence level IV; recommendation grade $\mathrm{C}$ ).

Screening with either EIA alone (evidence level IIb; recommendation grade $\mathrm{B}$ ) or the TPPA alone (evidence level $\mathrm{IV}$; recommendation grade $\mathrm{C}$ ) is recommended ${ }^{4}$ (the TPPA is more sensitive than the TPHA in primary infection ${ }^{5}$ ).

The TPHA can be used in combination with a cardiolipin antigen/reagin test such as VDRL or RPR to maximise the detection of primary infection on screening (evidence level III; recommendation grade B).

\section{Additional and confirmatory serological tests (evidence level IV; recommendation grade C)}

An EIA IgM test should be performed in addition to routine screening tests in all cases of genital ulceration as well as in those who are known contacts of syphilis (see below). ${ }^{4}$ Note: the rationale for this is that $\operatorname{IgM}$ becomes detectable in the serum 2-3 weeks after infection and IgG 4-5 weeks after infection. Therefore there will be a window of $1-2$ weeks when routine screening tests may be negative.

A quantitative TPPA should be used to confirm a positive EIA. $^{24}$

An EIA should be used to confirm a positive TPPA. ${ }^{24}$

An additional test such as immunoblotting based on recombinant antigens ${ }^{6}$ or the fluorescent antibody absorbed (FTA-abs) test $^{2}$ can be used in the case of a discrepancy between the EIA and TPPA.

An EIA for anti-treponemal IgM should be performed on all sera reactive in one or more of the screening tests. ${ }^{4}$

Quantitative VDRL/RPR tests should be performed before therapy. ${ }^{4}$

Note: in patients who have previously been treated for syphilis a fourfold increase in VDRL/RPR titre and/or a change in the EIA IgM from negative to positive (confirmed on a second specimen) suggests re-infection or relapse.

\section{Direct detection of $T$ pallidum in primary and secondary syphilis}

Dark ground/dark field (DGM) microscopy of lesion exudate or lymph nodes should be performed by experienced clinicians $^{7}$ (evidence level IV; recommendation grade C). Because of interference from commensal spirochaetes that are found in the normal flora of the genital and rectal mucosae, DGM is considered to be less reliable in examining rectal and non-penile genital lesions. DGM is not suitable for examining oral lesions.

Note: To obtain lesion exudates from a presumptive syphilitic chancre for DGM, the ulcer should be cleaned with sterile saline using a gauze swab. Any crust on the ulcer surface should first be removed. The ulcer should then be squeezed for long enough to produce sufficient serous fluid to be collected by a loop or other suitable instrument and placed on a glass microscope slide. The exudate should have a coverslip placed over it and DGM performed within 10 minutes in order to look for the characteristic morphology and motility of $T$ pallidum organisms. Other sites from which exudative material can be examined include skin lesions (after removal of the epithelial surface) and condylomata lata. Material from enlarged lymph nodes can be aspirated using a sterile 23 gauge needle and syringe filled with $0.2 \mathrm{ml}$ of sterile saline.

If the initial examination is negative DGM should be repeated daily for at least 3 days: antibiotics should be withheld during this period-local saline lavage may be used to reduce local sepsis (evidence level IV; recommendation grade $\mathrm{C})$.

Abbreviations: DGM, dark ground microscopy; EIA, enzyme immunoassay; FTA-abs test, fluorescent antibody absorbed test; GUM, genitourinary medicine; PCR, polymerase chain reaction; STI, sexually transmitted infections; TPHA, Treponema pallidum haemagglutination assay; TPPA, Treponema pallidum particle assay 
Testing of material submitted on dry swabs by the polymerase chain reaction (PCR) is recommended for oral or other lesions where contamination with commensal treponemes is likely ${ }^{78}$ (evidence level IV; recommendation grade $C$ ).

PCR is also useful in the diagnosis of primary syphilis and is available via local laboratories sending samples to the Sexually Transmitted Bacteria Reference Laboratory (STBRL) at the Health Protection Agency (stbrl@hpa.org.uk) (evidence level IV; recommendation grade C).

\section{RECOMMENDED SITES FOR TESTING}

- Clotted blood (all patients)

- Ulcer material (primary syphilis)

- Lesion material (secondary syphilis).

\section{FACTORS THAT ALTER TESTS RECOMMENDED OR SITES TESTED}

Genital or extragenital lesions (including oral) that could be caused by primary syphilis or a history of sexual contact with a patient known to have syphilis are the only factors that would influence the recommended tests or sites tested. In these circumstances an anti-treponemal IgM EIA should be performed in addition to the routine tests (see above).

Other aspects of sexual history (for example, oral sex, unprotected sex with multiple partners, past history of sexually transmitted disease, sexual assault) will not alter tests or sites but factors such as unprotected oral, vaginal or anal sex with multiple partners and sexual assault may influence the frequency of repeat testing (see below, Recommendation for frequency of repeat testing in an asymptomatic patient).

\section{Risk groups}

- MSM (no alteration to standard recommendation)

- Sex workers (no alteration to standard recommendation)

- "Young" (under 25) patients (no alteration to standard recommendation).

\section{Other groups}

- Pregnant women (no alteration to standard recommendation)

- Women with history of hysterectomy (no alteration to standard recommendation)

- Patients who are known contacts of the infection need a request for an anti-treponemal IgM EIA on the blood specimen submitted for standard screening. ${ }^{7}$

\section{RECOMMENDATION FOR FREQUENCY OF REPEAT TESTING IN AN ASYMPTOMATIC PATIENT (EVIDENCE LEVEL IV; RECOMMENDATION GRADE C IN EACH CASE)}

The frequency of repeat testing depends on the sexual history, particularly type of sexual exposure and number of sexual partners.

A "high risk" exposure would include unprotected oral, anal, or vaginal intercourse with a "high risk" partner-for example, partner with suspected or proved syphilis, homosexual male with multiple partners, anonymous partner(s) in saunas and other venues, commercial sex worker, partner just arrived from or living in a country where the prevalence of syphilis is known to be high.

No further testing is recommended if the patient had a single "low risk" episode more than 6 weeks previously (this is a pragmatic approach but is based on the scientific premise that the average pre-patent period is 3 weeks and IgG production starts around the fourth week of infection).

A repeat screening test is recommended 3 months after exposure if the patient had a single "high risk" exposure less than 6 weeks before attending the clinic.

Routine screening as well as specific EIA-IgM tests should be repeated at 6 weeks and 3 months for patients who:

- have had multiple "high risk" exposures

- have DGM negative ulcerative lesions that could be the result of primary syphilis

- are contacts of a suspected or proved case of syphilis, regardless of whether they have received epidemiological treatment for syphilis.

Patients with "high risk" exposures should be informed about the symptoms of primary or secondary syphilis and encouraged to return immediately if these develop before the next serological screening visit.

\section{RECOMMENDATION FOR TEST OF CURE}

- Quantitative VDRL/RPR tests are recommended (evidence level III; recommendation grade B) and should be performed with the same antigen (manufacturer) and in the same laboratory ${ }^{4}$ (evidence level IV; recommendation grade $\mathrm{C}$ ).

- VDRL/RPR tests should be performed monthly for 3 months and at 6 months and 12 months for early (infectious) syphilis ${ }^{79}$ (evidence level IV; recommendation grade $\mathrm{C}$ ).

- VDRL/RPR tests should be performed every 6 months until negative/serofast for late (non-infectious) syphilis ${ }^{9}$ (evidence level IV; recommendation grade C).

- HIV positive patients should have repeat treponemal serology performed yearly, or more frequently if at risk of re-infection with syphilis through their sexual activity (see above, Recommendations for frequency of repeat testing) (evidence level IV; recommendation grade C).

- Lumbar punctures are not normally taken in early syphilis. If lumbar puncture is taken in accordance with appropriate guidelines ${ }^{7}{ }^{10}$ then the CSF should be tested on a 6 monthly basis until the cell count is normal (evidence level IV; recommendation grade C).

\section{STAKEHOLDER INVOLVEMENT PHLS Syphilis Forum}

No patient involvement has been undertaken

\section{RIGOUR OF DEVELOPMENT}

This guideline was obtained by searching the Medline database from 1965 up until August 2002 using the MeSH headings "syphilis", "Treponema pallidum", "serodiagnosis". The recommendations of the PHLS Syphilis Forum, ${ }^{2}{ }^{4}$ the UK national guidelines for the management of syphilis, ${ }^{7}{ }^{10}$ the European guidelines for the management of syphilis, ${ }^{9}$ and the CDC STI treatment guidelines of $2002^{11}$ were used as a source for expert consensus.

A key review paper ${ }^{12}$ was also consulted.

\section{APPLICABILITY/RESOURCE REQUIREMENTS}

The guideline recommends the use of EIA IgM serological tests and PCR testing in certain situations. As these tests are not routinely available, this will impact on laboratory staff as samples, particularly for PCR, will need to be sent away to specialist or reference laboratories capable of performing these tests. 
Staff in GUM clinics will need to be trained in DGM to increase the sensitivity and the specificity of this test in routine clinical practice.

\section{AUDITABLE OUTCOME MEASURES}

(a) At least $90 \%$ of patients at "high risk" of syphilis should be re-screened on at least one occasion within 3 months of their first serological test.

(b) At least $80 \%$ of patients treated for syphilis should have a repeat VDRL/RPR within 3 months of treatment and at least $70 \%$ should return for a second VDRL/RPR around 6 months.

Comparison with the pretreatment titre should show the following:

- In primary and secondary syphilis, VDRL/RPR titres should decrease fourfold by 3-6 months and eightfold by 6-12 months. ${ }^{13}{ }^{14}$

- In early latent syphilis, VDRL/RPR titres should decrease fourfold by 12 months. ${ }^{13} 14$

- A fourfold increase in VDRL/RPR titre (confirmed on a second specimen) suggests re-infection or relapse. ${ }^{4}$

\author{
Authors' affiliations \\ D A Lewis, STI Reference Centre, National Institute for Communicable \\ Diseases, Johannesburg, South Africa \\ H Young, STD/Chlamydia Laboratory, Department of Laboratory \\ Medicine (Microbiology), Edinburgh Royal Infirmary, Edinburgh, UK \\ Conflicts of interest: none. \\ Correspondence to: Dr David A Lewis, STI Reference Centre, National \\ Institute for Communicable Diseases, Private Bag X4, Sandringham, \\ 2131, South Africa; davidl@nicd.ac.za
}

Accepted for publication 31 August 2006

\section{REFERENCES}

1 Nicoll A, Hamers FH. Are trends in HIV, gonorrhoea, and syphilis worsening in western Europe? BMJ 2002;324:1324-7

2 Egglestone SI. Turner AJL on behalf of the Syphilis Forum. Serological diagnosis of syphilis. Commun Dis Public Health 2000;3:158-62.

3 Schmidt BL, Edilalipour M, Luger A. Comparative evaluation of nine different enzyme-linked immunosorbent assays for determination of antibodies against Treponema pallidum in patients with primary syphilis. J Clin Microbiol 2000;38:1279-82.

4 Turner AJL, Egglestone SI, Young $\mathrm{H}$, on behalf of the Syphilis Forum. Diagnosis of syphilis: guidelines for the laboratory diagnosis in co-existing HIV infection, neurosyphilis, antenatal screening and congenital syphilis. (In preparation).

5 Kobayashi S, Yamaya S-I, Sugahara T, et al. Microcapsule agglutination tests for Treponema pallidum antibodies: a new serodiagnostic test for syphilis. Br J Vener Dis 1983;59:1-7.

6 Ebel A, Vanneste L, Cardinaels M, et al. Validation of the INNO-LIA syphilis kit as a confirmatory assay for Treponema pallidum antibodies. J Clin Microbiol 2000;38:215-9.

7 UK national guideline for the management of early syphilis BASHH Clinical Effectiveness Guidelines, 2002. http://www.bashh.org/guidelines/2002/ early \$final0502.pdf.

8 Orle KA, Gates CA, Martin DH, et al. Simultaneous PCR detection of Haemophilius ducreyi, Treponema pallidum, and herpes simplex virus types 1 and 2 from genital ulcers. J Clin Microbiol 1996;34:49-54.

9 Goh BT, vanVoorst Vader PC. European guidelines for the management of syphilis. Int J STD AIDS 2001;12(Suppl 3):14-26.

10 UK national guideline for the management of late syphilis BASHH Clinical Effectiveness Guidelines, 2002. http://www.bashh.org/guidelines/2002/ late\$final_b_311202.pdf.

11 Centers for Disease Control and Prevention. Sexually transmitted diseases treatment guidelines 2002. MMWR 2002;51(No RR-6):18-25.

12 Young H. Syphilis: new diagnostic directions. Int J STD AIDS 1992;3:391-413.

13 Brown ST, Zaidi A, Larsen SA, et al. Serological response to syphilis treatment. A new analysis of old data. JAMA 1985;253:1296-9.

14 Romanowski B, Sutherland R, Fick GH, et al. Serologic response to treatment of infectious syphilis. Ann Intern Med 1991;114:1005-9. 